\title{
Wireless sensor networks lifetime assessment model development
}

\author{
Aleksejs Jurenoks, Leonids Novickis \\ Riga Technical University, Riga, Latvia \\ Contactpoint: aleksejs.jurenoks@rtu.lv,lnovickis@gmail.com
}

\begin{abstract}
In the recent year's low power computing systems have gained popularity. Networks, which use low power computer systems and transmitted data by using wireless connection are called wireless sensor networks, which main task is to get the information from sensors and transmission network. Nowadays, the most topical researches pertaining to wireless sensor networks are grounded on the new optimization of structure of network transmission protocol, the routing optimization in transmission network, optimization of network structure, as a result of which the life circle of wireless network sensors is possible to increase. In the present article the methodology for determining the life circle of network is discussed. The approaches in detection of life circle pertaining to the important network nodes are described.
\end{abstract}

Keywords: Arduino; Sensor Network; life circle; Data processing.

\section{INTRODUCTION}

More institutions are concerned with the problems of processing real time data every day. The bigger the data flow, the longer the processing time is necessary. According to IDC data in year 2008 information data size of "digital universe" was 1,6 ZB and it will be increased at least twice to year 2015 .

In the recent years low power computing systems have gained great popularity. Nowadays, the technologies allow to decrease the existing size of the system creating an inexpensive low power computing systems with the ability to use both physical and wireless networks for transmission of information. The main popularity this type of technology has gained in the field of receiving information from sensors and control systems. Network that uses low power computing and transmitting the data via a wireless connection is referred to as wireless sensor networks, the main task of which is receiving information from the sensors and transmission in the network.

The main drawback of wireless sensor networks (BST) is the low power batteries which significantly limit the lifetime of the network.

Therefore, this article discusses the developed model of sensor network, which allows to assess the life circle of network by using the way of working of each network element. The methodology for the calculation of the parameters of the main model are described in details. The life circle of the separate network nodes is estimated by using the defined parameter values.

\section{RELATED WORKS}

Nowadays, the most topical researches pertaining to wireless sensor networks are grounded on the new optimization of structure of network transmission protocol, routing optimization in the transmission network, optimization of network structure, as a result of which the life circle of wireless network sensors is possible to increase.

In the network exists unequal power consumption; thus, the net nodes become disable at the moment when, despite the fact that a larger part of the network continues to operate, the electricity finishes in some network nodes. [2, 15]

There are a number of methods that allow to solve this problem $[11,13]$. These include the choice of the capacity of individual battery, the deployment of the node density, the adjustment of power transmitter, the application of energy-efficient data transfer protocol, positioning of network nodes and other methods that are associated with the introduction of an additional network costs. Relatively recently a new method for balancing energy through the mobility of network node, which provides the opportunity for reconfiguration of dynamic network or change of network topology, has been offered [14].

However, the main drawback of this method of study is absence of mathematical model of the dynamic network. 


\section{NETWORK LIFE EXPECTANCY VALUATION MODEL}

The life circle of network depends on how long its elements are working. Taking into account that verbosity is often included in networks, different requirements exist for the quality of the results received from the network.

While working out the network, it is necessary do timely assessment of the approximate working time pertaining to each network element until the moment when the battery change of the element will be necessary. It is important to understand what factors influence the autonomous working time of network elements.

It is well known that the power consumption of individual elements depends on several factors to consider when designing networks:

- The technical parameters of node;

- Frequency of data collection;

- Physical and channel level protocols;

- Network topology;

- Use of routing protocol.

Any sensor network has three types of nodes terminals, routers, and data collectors. Let us assume that the data collectors do not affect the life circle of the overall system because they are provided with independent power supply or are equipped with a much more powerful autonomous power supply. Currently, there is a variety of creators/indicators of network life circle.

\section{A. The Index Based on the Working Node}

The total time of network operation can be marked as $T_{k}^{n}$ time, in which at least $\mathrm{k}$ of the $\mathrm{n}$ units are in operation $[1,3,4,5]$. However, there is one drawback of this marking- the types of network nodes are not defined. Mostly in the networks there are primary nodes - which provide data retransmission and significantly impact the overall network performance. If one of the "bearing" nodes stops working, it automatically means that the network ceases to exist. That is why in many works [6,7], analyzing the life circle of system, variable $\mathrm{m}$, which means the number of critical important elements in the network, which must be active at all times, is defined. For example they could be the nodes of network cluster management [8]. For the other nodes, metrics is used:

$$
T_{k}^{n-m} \text {. }
$$

In the researches it is often used a case when $k=n$. In this case the network is considered as able-bodied as long as all the network nodes are active or:

$$
T_{n}^{n}=\min _{i \in V_{n}} T_{i}
$$

where: $V_{n}$ - the quantity of network nodes;

$T_{i}$ - the life circle of each network node.

\section{B. The Indicator Based on the Area Coverage}

This indicator is related to the use of network sensor- the quantity of information that is necessary to get from the concrete network segment. There are two approaches on the determination of the indicators based in the coverage. On the basis of the first approach the network is considered as able-bodied as long as $\alpha$ percent of the overall network coverage is covered by at least one sensor ( $\alpha$ - coverage) [9]. The second approach is based on provision of redundancy and requires that in each network segment should be at least $\mathrm{k}$ - number of active sensors ( $\mathrm{k}$ - coverage) [10].

The biggest disadvantage pertaining to the detection of indicators based coverage is the complex process of algorithmic presentation.

\section{Index Based on the Delay of Information Transmission}

According to the research, this index is mostly used when working with information gathering sensor network systems. The full definition of the index is provided in the paper [3]. Let us improve this index in order to cover previously mentioned index groups.

Suppose that the environment, which is carried out by the sensor network monitoring system, at each time interval occurs in certain activities which need to be identified. The events can be considered as external events, such as alerts, temperature changes of the system and other technical information, as well as described determinate event of the internal network, such as a regular transmission of information.

Let us introduce the parameters of quality pertaining to working network for each zone at specified time interval t. Suppose that $N_{k}(t)-$ in the range $\mathrm{k}$ there is the total amount of events in the time interval $\mathrm{t}$ and $I_{k}(t)$ - the total amount of the events from the $N_{k}(t)$ number that are delivered to a data collector at the determined period of time. The total time $\mathrm{t}$ is defined for the whole range $\mathrm{k}$. The parameters $\Delta t_{k}$ are defined on the basis of the necessary network intensity in the determined network segment. In the result zone at $\mathrm{k}$ time in the $\mathrm{t}$ interval the indicator of network working quality will be equal to:

$$
Q_{k}(t)=\left\{\begin{array}{ll}
\frac{I_{k}(t)}{N_{k}(t)}, & N_{k}(t) \neq 0 \\
1, & N_{k}(t)=0
\end{array} .\right.
$$

For acting network there is a threshold $c_{k}$ that exists in order to indicate the lowest ratio value $Q_{k}$ below which the network can not be regarded as working age. Thus, for the possible duration of the network can be considered:

$$
\forall t<\tau_{1}, \forall k \in[1 . . m]: Q_{k}(t) \geq C_{k} .
$$




\section{CALCULATION OF CONSUMED ENERGY PERTAINING TO THE NODES OF SENSOR NETWORK}

\section{A. The terminal lifespan assessment method}

The terminal node of the sensor network is intended to reading information from the sensors for the further transmission of data in the network.

The main difference in the node from the router is the impossibility of information transmission. This means that the terminal node does not use power and time for reception of information.

The consumed terminal energy in one operating cycle of the system can be defined by the following formula:

$$
P_{\text {term }}=P_{t x} * t_{t x}+P_{s} * t_{s}+P_{a} * t_{a},
$$

where $P_{t x}-$ the average conumed energy in the transmission mode (W);

$t_{t x}$ - time that is required for the information transmission in the network (s);

$P_{S}$ - average power consumed in a sleeping mode (W);

$t_{s}$ - time in which the system is in the sleeping mode (s);

$P_{a}-$ consumed power on processing of data (reading the information from sensors) (W);

$t_{a}$ - time that is intended for processing the information (s).

It is assumed that $t_{t x}+t_{a}>t_{s}$, which means that the system has sufficient time to be in the sleeping mode. The value of parameters is determined by the technical data of processor and properties of software algorithm.

For operating wireless network, it is important to deliver the data to the recipient node and, depending on the situation, the coefficient is impossible to define precisely.

There might appear a situation that the system will be unable to deliver the information to the recipient, and then it will be necessary to resent it as long as the information is delivered. As a result, the total sleeping time $\Delta t_{t x} \leq t_{s}$ will be decreased. Consequently, it means that the system can dedicate time for resending information no more than the amount of time $t_{s}$. If the information has not been delivered, it will be considered as lost.

\section{B. The router lifespan assessment method}

It is known that in sensor networks oriented on events, which are using asynchronous data access environment, a router is the weakest point regarding the duration of life circle of the system.

It is based on the fact that a node spends very little time or at all does not spend time in the sleeping mode, but consumes power on reception of information from the network and the transmission of information into network.
The total power consumption of the router very much depends on the selected routing protocol that in the end determines the table of service data flow for safety.

A router has four operating conditions:

$t_{s}$ - sleeping mode of the system;

$t_{s}$ - The information reception from a network;

$t_{t x}$ - Information sharing in a network;

$t_{a}$ - Reading the information from the sensors.

Since the performance of the network is highly determined, it can be considered that the router $\Delta t_{r x}+\Delta t_{t x} \leq t_{s}$. While transmitting the information, an error may appear in the network; consequently, it will take more time to send and receive data; as a result, it is necessary to introduce an additional parameter $p_{e}$ - percentage denoting error probability.

Using error probability coefficient in the network can be considered that:

$$
p_{e}\left(\Delta t_{r x}+\Delta t_{t x}\right) \leq t_{s}
$$

The amount of time the system spends on the reception of all the information is equal to:

$$
r_{r x}=\sum_{i=1}^{n} t_{i} * I_{i},
$$

where $t_{i}$ - the time necessary for the reception of information from one terminal (s);

$I_{i}$ - i-the intensity of data flow.

The amount of time that the system will spend on sending the information in the network is equal to:

$$
r_{t x}=\sum_{i=1}^{n+1} t_{i} * I_{i}
$$

There is a situation when the system is not able to transfer all the necessary information that has been received by the node $r_{r x}>r_{t x}$. Before the information is received the system checks whether there will be sufficient time for transferring the information.

When planning the router load, the total life cycle of the router $\Delta r$ must be taken into account.

$$
\Delta r<p_{e}\left(r_{r x}+r_{t x)}+r_{a}\right.
$$

where $r_{a}$-the time consumed by the system to receive information from the sensors (s).

Let us look at the situation $r_{r x}+r_{t x}+r_{a} \leq r_{s}$, when the router is able to fulfill its task and transfer the information to the network. In this case, the router's power consumption will be equal to:

$$
P_{m}=P_{a} r_{a}+P_{t x} r_{t x}+P_{r x} r_{r x}+\left(r_{s}-r_{t x}-r_{r s}\right) P_{s}
$$

where $P_{t x}-$ the average power of router in the transmission mode, $P_{r x}$ - the average power of router in the receiving mode, $P_{a}$ - the average power of router receiving information from sensors, $P_{s}$ - the power of router in the sleeping mode. 


\section{The Impact of Operating Range on the Work of the Terminal}

Each network element has defined range in which it can operate. The amount of the consumed energy on transmission of information is influenced by the distance to which the information has been transmitted. The router of the system and the terminal can operate in several modes that will reduce the amount of consumed energy by rationally deploying elements of the network, as well as determining the location of network elements.

Each transmitter is provided with optimal transmission distance, working in which power consumption is more efficient. The power of transmitter determines the speed of the transmission of information. In the study it is believed that the system is operating in the same environment, and the speed of information transmission is not affected externally.

It is known that the system consumes certain power for the transmission of data to the maximum distance [12]. Using the formula for determination of radio signal amplitude provided by Boris Vvedenskij, it is possible to determine the admissible maximum distance to which the information can be transmitted.

$$
E_{n p}=\frac{4 \pi \sqrt{P * G}}{\lambda * r^{2}} * h_{1} * h_{2},
$$

where

$E_{n p}$ - wave amplitude in the receiving point;

$P$ - power coefficient of retranslator;

$G$ - coefficient of wave amplifier;

$\lambda$ - wavelength;

$r$ - distance of radio communications;

$h_{1}, h_{2}$ - height to which radio antenna was lifted.

It is assumed that all network nodes in our system use antennas of the same type and do not use additional intensifiers; thus, the simplified formula can be used:

$$
E_{n p}=\frac{4 \pi \sqrt{P}}{\lambda * r^{2}} .
$$

Changing the distances of network node, it is important not to lose the quality of signal. It is assumed that in case of increasing the distance the $E_{n p}$ value will remain unchanged. In the result the $P_{k}$ coefficient of consumed power pertaining to router is equal to:

$$
P_{k}=\frac{E_{n p^{2} * \lambda^{2} * r^{4}}}{(4 \pi)^{2}},
$$

When planning the operation scheme of sensor network there is a possibility to equalize the consumed power of network element by adapting the power of transmitter and operating range to certain network segment. This approach will allow to equalize the total duration of life circle pertaining to nodes by decreasing the left out quantity of energy in the nodes.

As a result, the consumed power of router while transmitting the information in the network is equal to:

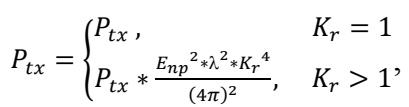

where $K_{r}$ - the coefficient of working range against standard working range.

\section{IEEE 802.15.4 Standard Frame Properties}

The maximum size of the load efficiency pertaining to frame is dependent on the length of service field. The standard describes the maximum length of the physical level of the frameaMaxPHYPacketSize $=$ 127 bytes. If to take into account the minimum size of addressing technical frame comprises 4 bytes, the maximum coefficient of efficiency load will be equal to 112 bytes.

IEEE 802.15.4 standard operates at $2.4 \mathrm{GHz}$ frequency with a permissible transfer rate of channel $f=250 \mathrm{kbit} / \mathrm{s}$. The result time required for transmission of information in the network can be calculated using the following formula:

$$
t_{D A T A}=\frac{L+O}{f},
$$

where $L$ - the number of bits per packet, $O$ - the size of the service field in bits.

\section{$\mathrm{V}$ THE PRACTICAL EXAMPLE OF CALCULATION OF POWER CONSUMPTION}

\section{A. The Terminal Node power consumption}

Let us assume that the frequency of device processor is $f_{\text {cpu }}$ : one activity takes $C$ numbers of processor cycles and it is necessary to execute $M$ number of measuring amount in order to secure the preparation of the information to be sent to the network. In case of recalculation, the time necessary for the transition form the sleeping mode $t_{s}$ must be taken into account.

The result time that is necessary for system to be in the active mode is equal to:

$$
t_{a}=\frac{C * M}{f_{c p u}}+t_{s},
$$

Let us assume that the system operates:

- using a single AA battery types - accordingly the initial energy $E_{0}=9 \mathrm{~kJ}$;

- Each operating cycle requires two CPU cycles $-C=18$;

- For the system to perform qualitative measurement 4 measurement cycles are needed $-M=4$ 
- The system uses low power processor $f_{c p u}=$ $16 \mathrm{MHz}$;

- The size of packet comprises 20 bytes $L=20$;

- The time that is required for the transition from the system sleep mode $-t_{s}=0.08 \mathrm{~s}$;

- The total time of system cycle $-t_{c}=20 \mathrm{~s}$.

Using the technical information of BSN node, let us calculate the consumed energy by one cycle of the terminal $\mathrm{mW}$.

$$
\begin{aligned}
& P_{\text {term }}=P_{t x} *\left(t_{\text {data }}+t_{A C L}\right)+P_{a} * t_{a}+P_{s} *\left(t_{c}-\right. \\
& \left.t_{\text {data }}-t_{A C L}-t_{a}\right) .
\end{aligned}
$$

The result is that, using output data, the terminal device for one cycle consumes $136.81 \mathrm{~mW}$. Knowing the $w$ - energy consumption of one time per 1 second and the $J$ - initial amount of energy let us calculate the total life circle of the system by using the following formula:

$$
s=\frac{J}{w} .
$$

\section{B. The Router Node power consumption}

Let us assume that the frequency of device processor is $f_{c p u}$ : one activity takes $C$ numbers of processor cycles and it is necessary to execute $M$ number of performances in order to accomplish the task. In case of recalculation, the time necessary for the transition from the sleeping mode must be taken into account $-t_{s}$.

The result time that is necessary for system to be in the active mode is equal to:

$$
t_{a}=\frac{C * M}{f_{c p u}}+t_{s} .
$$

The time that is necessary for receiving information from terminals is equal to:

$$
t_{r x}=\sum_{i=1}^{n} \frac{L}{f}+t_{D A T A} .
$$

where $t_{D A T A}$ for IEEE 802.15.4 with frame size of 11 byte $=0.352$.

The time that is necessary for sending information in the network is equal to:

$$
t_{t x}=\sum_{i=1}^{n+1} \frac{L}{f}+t_{D A T A} .
$$

Assume that the system works:

- using a single AA battery types - accordingly the initial energy $E_{0}=9 \mathrm{~kJ}$;

- the amount of processor cycles required for each working cycle: $C=18$;

- For the system to perform qualitative measurements 4 measurement cycles are needed $-M=4$;
- The system uses low power processor $f_{\text {сри }}=$ $16 \mathrm{MHz}$;

- The system uses the $2.4 \mathrm{GHz}$ frequency with the transmission rate $f=250 \mathrm{kbit} / \mathrm{s}$;

- The size of packet is 20 bytes $-L=20$;

- The time that is required for the transition from the system sleeping mode $t_{s}=0.08 \mathrm{~s}$;

- The total time of the cycle pertaining to the system $t_{c}=20 s$;

- The number of servicing terminals in the chain $-n=1$.

Using technical information, let us calculate the average power consumption in the router per time unit:

$$
P_{m}=\frac{P_{t x} t_{t x}+P_{r x} t_{r x}+P_{a} t_{a}+P_{s}\left(t_{c}-t_{t x}-t_{r x}-t_{a}\right)}{t_{c}} .
$$

The result is that, using output data, the terminal device for one cycle consumes $137.54 \mathrm{~mW}$. Knowing the energy consumption of one time per 1 second (w) and the initial amount of energy (J), let us calculate the total life circle of the system by using (17).

The results of the calculations show that on the same terms the energy of the router will expire faster than the energy of terminals. Furthermore, the bigger number is serviced by the router, the greater the energy balance remains in the terminal node.

\section{DEFINITION OF THE DURATION OF NETWORK}

In the process of operation, the network has the time when on the basis of external factors or lack of energy in various network elements system is unable to carry out its task and requires reconfiguration of the network.

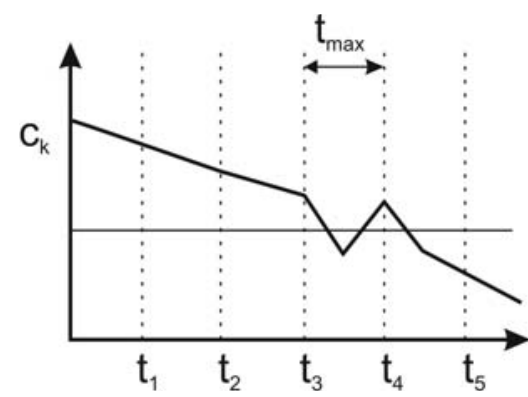

Fig. 1. Network reconfiguration delay time.

Let us define the number of points $\mathrm{N}$, which indicate the moment when the system crosses the border $-c_{k}$.

According to the drawing (Fig.1), the quantity is depicted by the points $t_{1}, t_{2}, . . t_{n}$. Suppose that there is the maximum allowable time $t_{\max }(\mathrm{s})$, when the system can be located below the range $c_{k}$ required for carrying out network reconfiguration. Overall system remains active if the following condition is fulfilled:

$$
t_{n+1}-t_{n}<t_{\max }
$$


By controlling the overall network management it is not possible in real time to find out the amount of energy left in nodes because it requires additional steps to transfer information. The only way to control the ability of life pertaining to the node is the delayed control while transmitting the information. If the time $\mathrm{T}$ of system reconfiguration is greater than $t_{\max }$, it is possible to verify the exit of an individual node from the rank and file.

This definition can be formalized by using the algorithm pertaining to the collection of information in the nodes (Fig.2.).

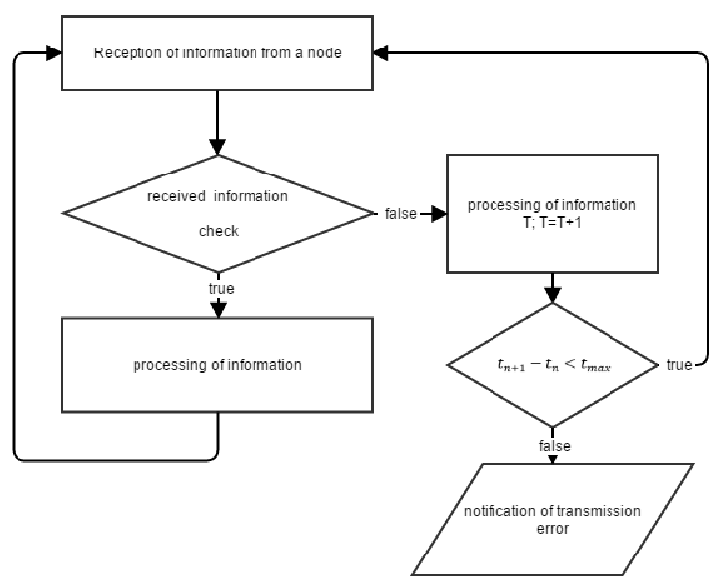

Fig.2. Information collection and processing algorithm.

\section{CONCLUSIONS}

The article describes the developed methodology for calculation of life circle pertaining to wireless sensor networks, which allows to evaluate the life circle of each network element and to determine the overall life circle of the network using the developed structure of network. Described methodology is design to fit any Wireless sensor network devices that are using IEEE 802.15.4 transmission protocols. Additional protocol changes structure need to be taken to use it with any $802 . x$ technology. This approach differs from the others with as follows:

- description of each element of network by using the quantity coefficient of the consumed power;

- using the coefficient of object-range transmission for calculation of power;

- uses the coefficient of changes of network configuration and determines the denial of time pertaining to the system.

It is known that the total amount of consumed energy in the nodes depends on many factors:

- technical solutions of hardware;

- frequency of operation/performance;

- intensity of data flow;

- algorithms for access to the environment.

In the article the developed methodology allows efficient planning of the structure pertaining to the network by dividing the tasks between the network nodes, thus increasing the overall life circle of network.

In further article we will describe wireless sensor network reconfiguration method that contributes to remaining energy balancing for network nodes.

\section{REFERENCES}

[1] Chen B., Jamieson K., Balakrishnan H., Morris R. Span: An energy-efficient coordination algorithm for topology maintenance in ad hoc wireless networks / ACM Wireless Networks Journal. 2001. P. 85-96.

[2] Chen D., Varshney P. K. QoS Support in Wireless Sensor Networks: A Survey / Proc. of the 2004 International Conference on Wireless Networks (ICWN 2004), Las Vegas, Nevada, USA. 2004. P. 227-233.

[3] Chen Y., Zhao Q. On the lifetime of wireless sensor networks/ Communications Letters, IEEE. Nov. Vol.9, no.11. P.976978.

[4] Hellman K., Colagrosso M. Investigating a Wireless Sensor Network Optimal Lifetime Solution for Linear Topologies / Journal of Interconnection Networks. 2006. P. 91-100.

[5] Kang I., Poovendran R. Maximizing static network lifetime of wireless broadcast ad hoc networks / Communications, 2003. ICC '03. IEEE International Conference on. Vol. 3. May. P. 2256-2261.

[6] Kumar S., Lai T. H., Balogh J. On k-coverage in a mostly sleeping sensor network / Proceedings of the 10th annual international conference on Mobile computing and networking. MobiCom '04. New York, NY, USA: ACM, 2004. P. 144-158.

[7] Lu G., Krishnamachari B., Raghavendra C. An adaptive energy-efficient and low-latency MAC for data gathering in wireless sensor networks / Parallel and Distributed Processing Symposium, 2004. Proceedings. 18th International. 2004. P. 224-231.

[8] Raicu I., Schwiebert L., Fowler S., Gupta E. K. S. Local load balancing for globally efficient routing in wireless sensor networks / International Journal of Distributed Sensor Networks. 2005. Vol. 1. P. 2005.

[9] Soro S., Heinzelman W. B. Prolonging the lifetime of wireless sensor networks via unequal clustering / Proceedings of the 19th IEEE International Parallel and Distributed Processing Symposium (IPDPS'05). IPDPS '05. Washington, DC, USA: IEEE Computer Society, 2005. P. 236-243.

[10] Vass D., Vincze Z., Vida R., Vidacs A. Energy Efficiency in Wireless Sensor Networks Using Mobile Base Station / EUNICE 2005: Networks and Applications Towards a Ubiquitously Connected World / Ed. by C. Kloos, A. Marin, D. Larrabeiti. Springer US, 2006. Vol. 196 of IFIP ternational Federation for Information Processing. P. 173-186.

[11] Vullers R., van Schaijk R., Doms I. et al. Micropower energy harvesting / Solid-State Electronics. 2009. Vol. 53, no. 7. P. $684-693$.

[12] Wu K., Gao Y., Li F., Xiao Y. Lightweight deployment-aware scheduling for wireless sensor networks / Mob. Netw. Appl. 2005. Vol. 10, no. 6. P. 837-852.

[13] Zhang H., Shen H. Balancing Energy Consumption to Maximize Network Lifetime in Data-Gathering Sensor Networks / IEEE Trans. Parallel Distrib. Syst. 2009. Vol. 20, no. 10. P. 1526-1539.

[14] Ефремов В. В., Маркман Г. 3. "Энергосбережение"и "энергоэффективность": уточнение понятий, система сбалансированных показателей энергоэффективности / Известия Томского политехнического университета. 2007. Т. 311, № 4. C. 146-148.

[15] Energy Harvesting Microstrain solutions URL (02.2015): http:/www.microstrain.com/energy-harvesting. 